\title{
Elaboração de um guia de atendimento a pacientes com crise hipertensiva para enfermeiros
}

\author{
Marcel Batista Botossi*, Carolina Vivian Gianvecchio**, Regimar Carla Machado, D.Sc.***
}

*Enfermeiro, Especialista em Enfermagem em Cuidados Críticos/Cardiologia da Universidade do Vale do Paraíba, São José dos Campos/SP, **Enfermeira, Docente do Curso de Pós-Graduação de Enfermagem em Cuidados Críticos/Cardiologia da Universidade do Vale do Paraíba, Especialista em Cuidados Criticos/Cardiologia da Universidade do Vale do Paraíba, Sáo José dos Campos/SP, ***Enfermeira, Docente do Departamento de Enfermagem da Universidade Federal do Rio Grande do Norte, Natal/RN

\section{Resumo}

Objetivo: Averiguar o conhecimento dos enfermeiros sobre o processo de atendimento a urgência e emergência hipertensiva e criar um guia de orientaçóes para a assistência do enfermeiro a pacientes com crise hipertensiva. Método: Estudo descritivo, exploratório, com uma abordagem quantitativa. Para a coleta de dados foi utilizado um instrumento embasado na revisão da literatura e em uma pesquisa, posteriormente sendo submetido ao ajuizamento por expertos. A amostra constituiu-se de 20 enfermeiros. Resultados: Observou-se que 60\% dos enfermeiros apresentaram desconhecimento sobre a definição de urgência hipertensiva e alteraçóes vasculares de maior incidência nos casos de crise hipertensiva. Conclusão: Os resultados demonstraram que os enfermeiros desconhecem questóes importantes sobre a crise hipertensiva afirmando assim a necessidade da criação de um guia de orientaçóes aos enfermeiros na abordagem dos pacientes em crise hipertensiva.

Palavras-chave: hipertensão, emergências, orientação, Enfermagem.

\section{Abstract}

\section{Preparation of a guide for nurses to care patients with hypertensive crisis}

Objective: To evaluate nurses' knowledge about the process of urgency and hypertensive emergency care and create a guide to help nurses caring for patients with hypertensive crisis. Methods: This is a descriptive and exploratory study, with a quantitative approach. We used an instrument based on literature review and a research which was subsequently subjected to expert judgment. The sample consisted of 20 nurses. Results: The definition of hypertensive emergency and most common vascular changes in patients with hypertensive crises were unknown by $60 \%$ of nurses. Conclusion: The results showed that nurses did not know about the important issues regarding hypertensive crisis, thus, we highlight the need of creating a guide to help nurses to manage patients in hypertensive crisis.

Key-words: hypertension, emergencies, orientation, Nursing. 


\section{Resumen}

\section{Elaboración de una guía de atención a pacientes con crisis hipertensiva para los enfermeros}

Objetivo: Evaluar los conocimientos de los enfermeros sobre el proceso de atención en urgencia y emergencia hipertensiva y crear una guía de orientación de cuidados para los enfermeros que tratan pacientes con crisis hipertensiva. Métodos: Estudio descriptivo, exploratorio, con abordaje cuantitativo. Para recolectar los datos, se utilizó un instrumento basado en la revisión de la literatura y una investigación que posteriormente fue sometido a juicio de expertos. El muestreo se constituyó de 20 enfermeros. Resultados: Se detectó que 60\% de los enfermeros desconocen la definición de urgencia hipertensiva y de alteraciones vasculares de mayor incidencia en pacientes con crisis hipertensivas. Conclusión: Los resultados mostraron que los enfermeros desconocen temas importantes sobre crisis hipertensiva reforzamos, así, la necesidad de crear una guía de orientación al personal de enfermería para el manejo de pacientes con crisis hipertensiva.

Palabras-clave: hipertensión, urgencias médicas, orientación, Enfermería.

\section{Introdução}

As doenças cardiovasculares são as que mais causam morte em todo o mundo, ocupando a primeira posição no perfil de morbimortalidade. Seu principal fator de risco é a Hipertensão Arterial Sistêmica (HAS) que vem atingindo grande parte da população do mundo $[1,2]$.

Uma das formas de apresentação ou mesmo de complicação da hipertensão arterial é a crise hipertensiva caracterizada pela presença de níveis da pressão arterial muito elevado, intenso, com sintomas e com risco de deterioração de órgãos alvos como o rim, cérebro, coração, retina e vasos sanguíneos. $\mathrm{O}$ tratamento incorreto leva ao risco de morte iminente ou potencial [3].

A crise hipertensiva pode ser classificada de acordo com as ocorrências clínicas, sendo elas emergências hipertensivas e urgências hipertensivas definidas como um grupo heterogêneo de doenças agudas $[4,5]$.

Nas urgências hipertensivas ocorre risco, mas sem sinais agudos de lesão de órgãos alvos e o aumento da pressão arterial está relacionada a sintomas como cefaléia, vertigem, agitação psicomotora e epistaxe. A pressão arterial (PA), nesses casos pode ser reduzida mais gradualmente, em questão de horas [6].

As emergências hipertensivas caracterizam-se pela pressão arterial muito elevada, proporcionando lesóes em órgãos alvos. Contudo, PA deve ser reduzida em questáo de minutos. A sintomatologia vai depender do órgão-alvo afetado e do grau de desenvolvimento dessa lesão [6].
Como a emergência hipertensiva requer cuidados imediatos dos profissionais de saúde e é uma rotina dos centros de emergência clínica, a presença do enfermeiro nestes locais torna-se imprescindível, desempenhando um papel de relevância nas relaçóes da equipe de saúde e exercendo uma forte ligação com a execução das atividades emergenciais $[7,8]$.

Todavia, não somente nas emergências hipertensivas, mas em quaisquer outras emergências, em muitos locais, permanece certo o despreparo profissional dos enfermeiros, acerca de atendimentos de emergência. Estudos mostram que muitos profissionais de saúde não estão registrando informaçôes importantes sobre o acompanhamento dos pacientes hipertensos e isso dificulta a análise da evoluçáo do tratamento, seja ele medicamentoso ou apenas mudança no estilo de vida [7,9].

Existem também muitas dificuldades encontradas entre os profissionais de saúde relacionadas principalmente a diferença de urgência e emergência hipertensiva. Neste contexto, este estudo visa averiguar o conhecimento dos enfermeiros sobre o processo de atendimento a urgência e emergência hipertensiva e criar um guia de orientaçóes para a assistência a pacientes em crise hipertensiva.

\section{Material e métodos}

Trata-se de um estudo com abordagem quantitativa, exploratória e desenvolvimento de um guia de orientações para enfermeiros.

Para o levantamento das dúvidas pertinentes a este estudo, foi construído um instrumento de coleta de dados totalmente estruturado, com questóes de 
múltipla escolha embasado na literatura científica $[6,7,9,10]$ e nas Diretrizes Brasileiras de Hipertensão 2010 [11].

Para a validação do conteúdo foi realizado o ajuizamento por cinco avaliadores médicos e enfermeiros considerados peritos no tema abordado com a finalidade de refinar o instrumento quanto à abrangência, à clareza e à pertinência. Optou-se pelo número cinco de avaliadores para evitar o empate de opinióes. Os peritos foram contatados primeiramente por e-mail e depois os instrumentos foram entregues pelo pesquisador. Após a conclusão da análise, foram realizadas alteraçóes de algumas variáveis do instrumento de acordo com as sugestóes dos avaliadores com a intenção de alcançar os objetivos do presente estudo.

O instrumento possuía 11 perguntas divididas em duas categorias: dados sociodemográficos dos participantes da pesquisa referentes ao tempo de formação profissional, atuaçáo na unidade de terapia intensiva e a fatores inerentes ao conhecimento do enfermeiro em relação ao atendimento a pacientes com crise hipertensiva, com alternativas fornecendo livre opção de resposta ao profissional participante.

$\mathrm{O}$ projeto de pesquisa foi submetido ao Comitê de Ética de Pesquisa (CEP) do Hospital Municipal José de Carvalho Florence, em São José dos Campos e aprovado sob o parecer no $024 / 12$ CEP/2012 conforme a Resolução 196/96 do Conselho Nacional de Saúde.

A amostra constituiu-se de 20 enfermeiros de um curso de Especializaçáo em enfermagem de uma Universidade no interior do Vale do Paraíba Paulista. Estabeleceu como critérios de inclusão os enfermeiros que aceitaram participar da pesquisa assinando o Termo de Consentimento Livre e Esclarecido. Portanto, não houve rejeição na participação do estudo.

Para a aplicação do instrumento de coleta dos dados, foi utilizada a sala de aula da Universidade e aplicado o instrumento aos enfermeiros no intervalo de aulas dos professores. $\mathrm{O}$ pesquisador permaneceu no local, para esclarecer dúvidas sobre as perguntas caso fosse necessário.

A análise das respostas, para fins de caracterizaçáo dos mesmos, foi realizada considerando-se os percentuais referentes aos respondentes (\% válido), excluindo-se aqueles enfermeiros que não se enquadravam nos critérios de inclusão do presente estudo, para obter um perfil real da amostra estudada.
Os dados obtidos foram transferidos para uma planilha e tabulados com o auxílio dos programas Microsoft Excel e Microsoft Word versóes do Office 2003. Para a confecção das tabelas, utilizou-se de números absolutos e porcentagens.

O critério utilizado para a formulação do guia de orientaçôes para assistência do enfermeiro a pacientes com crise hipertensiva foi baseado no desconhecimento dos enfermeiros perante as respostas de cada variável aplicada no instrumento de coleta de dados. Foram incluídas no guia as respostas às questôes com menos de $50 \%$ de acertos.

\section{Resultados}

Em relação aos dados sociodemográficos da amostra pesquisada houve um predomínio do sexo feminino 70,0\% (14), com uma prevalência de idade de 26 a 30 anos 50\% (10). No que diz respeito ao tempo de atividade profissional na UTI, verificou-se que $40,0 \%$ (8) atuam na unidade e quanto ao tempo exercido, $20,0 \%$ (4) com 1 a 2 anos de trabalho.

Tabela I - Distribuição dos enfermeiros que responderam as questões referentes à definição de emergência hipertensiva. São José dos Campos/SP 2012 ( $n=20$ ).

\begin{tabular}{|c|c|c|}
\hline $\begin{array}{l}\text { Domínio sobre a definição } \\
\text { de emergência hipertensiva }\end{array}$ & $\mathrm{N}$ & $\%$ \\
\hline $\begin{array}{l}\text { Risco iminente de morte ou deterioriza- } \\
\text { ção rápida de órgão alvo. Requer in- } \\
\text { tervenção imediata da pressão arterial, } \\
\text { avaliada em minutos ou algumas horas. }\end{array}$ & 17 & 85,00 \\
\hline $\begin{array}{l}\text { Risco iminente de morte ou deterioriza- } \\
\text { ção rápida de órgão alvo. Não requer } \\
\text { intervenção imediata da pressão arte- } \\
\text { rial, avaliada em algumas horas. }\end{array}$ & 0 & 0 \\
\hline $\begin{array}{l}\text { Risco iminente de morte ou deterioriza- } \\
\text { ção rápida de órgão alvo. Requer in- } \\
\text { tervenção imediata da pressão arterial, } \\
\text { avaliada em algumas horas. }\end{array}$ & 3 & 15,00 \\
\hline Total & 20 & 100,00 \\
\hline
\end{tabular}

Tabela II - Distribuição dos enfermeiros que responderam as questões referentes à definição de urgência hipertensiva. São José dos Campos/SP $(n=20)$.

\begin{tabular}{lll}
\hline \multicolumn{1}{c}{$\begin{array}{c}\text { Domínio sobre a definição } \\
\text { de urgência hipertensiva }\end{array}$} & N & $\%$ \\
\hline $\begin{array}{ll}\text { Risco menor de deteriorização de órgão } \\
\text { alvo. Risco potencial de morte. Redução } \\
\text { mais lenta da pressão arterial avaliada }\end{array}$ & 40,00 \\
em até 6 horas. & \\
\hline
\end{tabular}


Risco maior de deteriorização de órgão alvo. Risco potencial de morte. Redução mais lenta da pressão arterial avaliada em até 24 horas.

Risco menor de deteriorização de órgão alvo. Risco potencial de morte. Redução mais lenta da pressão arterial avaliada $5 \quad 25,00$ em até 24 horas

\begin{tabular}{lll}
\hline Total & 20 & 100,00 \\
\hline
\end{tabular}

Tabela III - Distribuição dos enfermeiros segundo a sequência utilizada para abordagem propedêutica do suporte diagnóstico ideais na emergência hipertensiva.

São José dos Campos/SP ( $\mathrm{n}=20$ ).

\begin{tabular}{lcc}
\hline $\begin{array}{c}\text { Domínio da abordagem propedêutica - } \\
\text { emergências hipertensivas }\end{array}$ & $\mathrm{N}$ & $\%$ \\
\hline $\begin{array}{l}\text { Entrevista, exame físico e exames com- } \\
\text { plementares }\end{array}$ & 18 & 90,00 \\
\hline Entrevista e exames complementares & 1 & 5,00 \\
\hline Exame físico e entrevista & 1 & 5,00 \\
\hline Total & 20 & 100,00 \\
\hline
\end{tabular}

Tabela IV - Distribuição dos enfermeiros que responderam as questões referentes às drogas hipotensoras indicadas nas emergências hipertensivas. São José dos Campos/SP ( $\mathrm{n}=20$ ).

\begin{tabular}{|c|c|c|}
\hline $\begin{array}{l}\text { Domínio sobre as drogas hipotensoras } \\
\text { indicadas nas emergências hipertensivas }\end{array}$ & $\mathrm{N}$ & $\%$ \\
\hline $\begin{array}{l}\text { Vasodilatadores, diurético, inibidores } \\
\text { adrenérgico }\end{array}$ & 10 & 50,00 \\
\hline $\begin{array}{l}\text { Drogas de uso oral, sublingual ou depen- } \\
\text { dendo da situação, drogas endovenosas }\end{array}$ & 9 & 45,00 \\
\hline Somente vasodilatadores & 1 & 5,00 \\
\hline Total & 20 & 100,00 \\
\hline
\end{tabular}

Tabela V - Distribuição dos enfermeiros que responderam as questões referentes às alterações cardiológicas mais frequentes nas emergências hipertensivas. São José dos Campos/SP ( $\mathrm{n}=20$ ).

\section{Domínio sobre as alterações}

cardiológicas mais frequentes nas $\quad N \quad \%$
emergências hipertensivas

Edema agudo de pulmão, insuficiência cardíaca refratária, infarto do miocárdio, crise adrenérgica.

Insuficiência cardíaca refratária, infarto agudo do miocárdio e crise adrenérgica.

210,00

Edema agudo de pulmão, insuficiência cardíaca refratária, infarto agudo do 1260,00 miocárdio.

$$
\text { Total }
$$

$20 \quad 100,00$

Tabela VI - Distribuição dos enfermeiros que responderam as questões referentes as alterações vasculares mais frequentes nas emergências hipertensivas. São José dos Campos/SP ( $\mathrm{n}=20$ ).

\begin{tabular}{lcc}
\hline $\begin{array}{c}\text { Domínio sobre as alterações vasculares } \\
\text { mais frequentes nas emergências } \\
\text { hipertensivas }\end{array}$ & N & $\%$ \\
\hline Dissecção aórtica & 8 & 40,00 \\
Estenose de artéria coronária & 7 & 35,00 \\
Formação de pseudoneurisma & 5 & 25,00 \\
\hline Total & 20 & 100,00 \\
\hline
\end{tabular}

\section{Discussão}

Com o aumento da expectativa de vida e o fenômeno do envelhecimento da população, algumas doenças têm aumentado a sua incidência e prevalência. A hipertensão arterial é a doença cardiovascular que mais aumentou nos últimos anos, atingido não só a população brasileira, mas toda a população mundial [11].

Neste contexto a assistência ao paciente com hipertensão arterial deve ser uma abordagem multidisciplinar, ou seja, uma ação integrada de uma gama de profissionais, tanto na assistência preventiva como intervencionista. Sobretudo, a enfermagem tem um papel fundamental, pois atua em diversas áreas junto aos pacientes hipertensos.

No entanto, o estudo demonstrou que na questão referente à definição de urgência hipertensiva, $40,0 \%$ apenas dos enfermeiros responderam corretamente, demonstrando uma deficiência no conhecimento sobre a temática. Este achado é semelhante a um estudo [10] em que $12 \%$ dos sujeitos participantes da pesquisa, responderam corretamente a definição de urgência hipertensiva.

A literatura define urgência hipertensiva com pacientes com aumento da pressão arterial sem risco ou pequeno risco de morte, e potencial para deteriorização do órgão-alvo [12]. O tratamento pode ser lento, em até 24 horas.

Define-se a emergência hipertensiva como a elevação da pressão arterial com risco efetivo de vida elou deterioração de órgão-alvo, sendo necessária a intervenção imediata para evitar complicaçóes irreversíveis [12].

Portanto, referente ao conhecimento sobre a definição de emergência hipertensiva, 85,0\% dos participantes desta pesquisa responderam corretamente. Contudo, é fundamental a importância entre as duas definiçóes, urgência e emergência 
hipertensiva para a indicação da terapêutica correta, pois muitos pacientes são medicados de maneira incorreta devido à conceituaçấo errada, podendo levar a efeitos colaterais indesejáveis [13].

$\mathrm{Na}$ questão sobre qual é a abordagem propedêutica e suporte diagnóstico realizado nas emergências hipertensivas, $90 \%$ dos enfermeiros responderam corretamente que é a entrevista, exame físico e exames complementares. A abordagem terapêutica deve ocorrer imediatamente devido à gravidade e ao risco de morte. $\mathrm{O}$ enfermeiro deve ter o domínio sobre tal procedimento que deve ser feito com rapidez, pois diante de uma emergência hipertensiva, a internação deve ser imediata, a fim de minimizar as lesóes em órgáos alvo [14,15].

Quando questionado sobre quais drogas hipotensoras estão indicadas para as emergências hipertensivas, a metade errou, pois somente 50\% dos enfermeiros responderam corretamente que são vasodilatadores, diuréticos, inibidores adrenérgicos. Essas drogas de ação curta, administrada por via endovenosa é a melhor escolha para o tratamento. O nitroprussiato de sódio é o vasodilatador mais utilizado nas situaçóes de emergências clínicas entre as drogas de escolha na abordagem inicial, ou seja, é recomendado como droga de primeira escolha para o tratamento das emergências hipertensivas, principalmente a encefalopatia hipertensiva e o edema agudo de pulmáo. Diuréticos podem ser utilizados nas emergências hipertensivas, principalmente naquelas que cursam com sobrecarga hídrica, manifestando-se sob a forma de congestão pulmonar e edema. Inibidores de enzima de conversão (IECA) também apresentam ampla indicação para o tratamento de crises hipertensivas $[10,14,16]$.

Referente a quais situações cardiológicas mais ocorridas nas emergências hipertensivas, $60 \%$ enfermeiros responderam corretamente que são edema agudo de pulmão, insuficiência cardíaca refratária e infarto agudo no miocárdio. Esses números são preocupantes devido a estas alteraçóes cardiológicas serem frequentes nos setores emergenciais. Estudos revelaram que neste país cerca de 13 milhóes de brasileiros apresentam hipertensão, aumentando, assim, a incidência de doenças como as complicaçóes cardiovasculares, podendo gerar doença cerebrovasculares, insuficiência cardíaca, renal e a emergência hipertensiva [17].

Quando questionados sobre quais as situaçóes vasculares mais ocorridas nas emergências hipertensivas, somente $40 \%$ enfermeiros responderam corretamente que é a dissecção aórtica. A emergência hipertensiva é um fator que predispóe a dissecção aórtica e sua manifestação atinge alto índice de mortalidade, em que a maioria dos casos localiza-se na aorta ascendente acometendo mais frequentemente homens [18].

Os níveis pressóricos elevados que estabelece a hipertensão arterial sistêmica constitui o principal fator de risco para a emergência hipertensiva. $O$ profissional envolvido com essa temática é o enfermeiro que desenvolve suas açóes individualmente através da consulta de enfermagem ou com grupos. Estudo mostra que a prevenção e/ou controle da pressão arterial é uma aproximação sistemática do relacionamento entre os enfermeiros e o paciente que, por meio da consulta de enfermagem tem influenciado fortemente no seguimento terapêutico e associa-se à maior adesão do tratamento $[9,19]$.

Há muito a ser pesquisado sobre a emergência hipertensiva no âmbito da enfermagem. A assistência a esses pacientes deve ser direcionada, com educação continuada considerando-se as particularidades de cada situação.

\section{Conclusão}

Os enfermeiros da amostra deste estudo apresentaram desconhecimento em variáveis como as situaçóes vasculares mais ocorridas nas emergências hipertensivas. Outra questão desconhecida pelos enfermeiros é quanto às drogas hipotensoras indicadas nas emergências hipertensivas. Além disso, também não sabiam o conceito de urgências hipertensivas.

Diante do exposto, criou-se um guia de orientaçáo aos enfermeiros, tendo como critério utilizado para a sua formulação o desconhecimento perante as variáveis aplicadas na pesquisa e outras questóes consideradas relevantes perante a literatura científica que contempla a temática ao qual se embasou o estudo.

\section{Referências}

1. Silva SSBE, Colósimo FC, Pierin AMG. The effect of educational interventions on nursing team knowledge about arterial hypertension. Rev Esc Enferm USP 2010;4(2):482-9.

2. Nogueira D, Faerstein E, Coeli CM, Chor D, Lopes CS, Wernek GL. Reconhecimento, tratamento e controle da hipertensão arterial: Estudo Pró-Saúde, Brasil. Rev Panam Salud Pública 2010;27(2):103-9.

3. Souza ACC, Moreira TMM, Silva MRF, Almeida PC. Acesso ao serviço de emergência pelos usuários com 
crise hipertensiva em um hospital de Fortaleza, CE, Brasil. Rev Bras Enferm 2009;62(4):535-9.

4. Born BJH, Beutle JJ, Gaillard CAJM, Gooijer A, Meiracker AH, Kroon AA. Dutch guideline for the management of hypertensive crisis -2010 . Neth J Med 2011;69(5):248-55.

5. Kazerani H, Hajimoradi B, Amini A, Naseri MH, Moharamzad Y. Clinical efficacy of sublingual captopril in the treatment of hypertensive urgency. Singap Med J 2009;50(4):400-2.

6. Amaro DSM, Machado RC, Silva MCF. Perfil de pacientes hipertensos: aspectos biossociais, antecedentes pessoais e tratamentos. Rev Enferm UFPE Online 2012;6(4):714-9.

7. Clark CE, Taylor RS, Shore AC, Ukoumunne OC, Campbell JL. Association of a difference in systolic blood pressure between arms with vascular disease and mortality: a systematic review and meta-analysis. Lancet 2012;379:905-14.

8. Oliveira M, Trindade MF. Atendimento de urgência e emergência na rede de atençáo básica de saúde: Análise do papel do enfermeiro e o processo de acolhimento. Revista Hórus 2010;2(4):160-71.

9. Maciel ICF, Araújo TL. Consulta de enfermagem: análise das açôes junto a programas de hipertensão arterial, em Fortaleza. Rev Latinoam Enferm 2003;11(2):20714.

10. Capucho LOH. Conhecimento dos enfermeiros sobre emergências hipertensivas que atuam em pronto socorro e unidade de terapia intensiva [Monografia]. São José dos Campos: UNIVAP; 2008.
11. Andrade JP, Nobre F, Tavares A, Brandáo AA, Sanjuliani AF, Nogueira AR, et al. VI Diretrizes Brasileiras de Hipertensão. Arq Bras Cardiol 2010;95(1 supl.1):1-51.

12. Sousa MG, Passarelli Jr O. Fluxograma de avaliação da hipertensão arterial resistente. Rev Factores de Risco 2013;30:21-3.

13. Nobre F, Chauchar F, Viana JM, Pereira GJV, Lima NKC. Avaliação do atendimento do hipertenso em serviço de urgência e em ambulatório de hipertenso. Rev Bras Cardiol 2002;78(2):156-8.

14. Araujo HBN, Fagundes AAP, Leite LR, Fonseca AGT. $\mathrm{O}$ uso de clevidipina em emergência hipertensiva. Rev Bras Ter Intensiva 2010;22(1):92-5.

15. Lesting JFP, Dias MA. Tratamento das crises hipertensivas. Perspect Medicas 2007;18(2):29-36.

16. Martin JFV, Loureiro AAC, Cipullo JP. Crise hipertensiva: atualização clínico-terapêutica. Arq Ciênc Saúde 2004;11(4):253-61.

17. Correia JN, Oliveira MZ. Avaliação do risco de acidente vascular cerebral em pacientes com hipertensão arterial sistêmica. Ciência et Práxis 2011;4(7):21-6.

18. Melo ROV, Toledo JCY, Braile DM. Dissecção aguda de aorta como apresentaçáo de emergência hipertensiva. Rev Bras Cir Cardiovasc 2008;23(4):586-8.

19. Borges JWP, Pinheiro NMG, Souza ACC. Hipertensão comunicada e hipertensão compreendida: saberes e práticas de enfermagem em um Programa de Saúde da Família de Fortaleza, Ceará. Ciênc Saúde Coletiva 2012;17(1):179-89.

\section{Guia de informações a enfermeiros sobre crises hipertensivas}

Classificação da pressão arterial de acordo com a medida casual no consultório (> 18 anos)

\begin{tabular}{|l|c|c|}
\hline \multicolumn{1}{|c|}{ Classificação } & $\begin{array}{c}\text { Pressão sistólica } \\
\mathrm{Mm} / \mathrm{hg}\end{array}$ & $\begin{array}{c}\text { Pressão diastólica } \\
\mathrm{Mm} / \mathrm{hg}\end{array}$ \\
\hline Ótima & $<120$ & $<80$ \\
\hline Normal & $<130$ & $<85$ \\
\hline Limítrofe & $130-139$ & $85-89$ \\
\hline Hipertensão estágio 1 & $140-159$ & $90-99$ \\
\hline Hipertensão estágio 2 & $160-179$ & $100-109$ \\
\hline Hipertensão estágio 3 & $\geq 180$ & $<110$ \\
\hline Hipertensão sistólica isolada & $\geq 140$ & $<90$ \\
\hline
\end{tabular}

Definição de urgência e emergência hipertensiva

\begin{tabular}{|l|l|}
\hline \multicolumn{1}{|c|}{ Emergência } & \multicolumn{1}{|c|}{ Urgência } \\
\hline Têm seu aumento da pressão arterial mais elevado. & Têm seu aumento da pressão arterial mais elevado. \\
\hline $\begin{array}{l}\text { Ocorre risco iminente de morte ou deteriorização rápida } \\
\text { de órgão alvo. }\end{array}$ & \begin{tabular}{l} 
Risco potencial de morte. \\
\hline $\begin{array}{l}\text { A sintomatologia vai depender do órgão-alvo afetado e do } \\
\text { grau de desenvolvimento dessa lesão. }\end{array}$
\end{tabular} \\
\hline $\begin{array}{l}\text { Requer intervenção imediata da pressão arterial, avaliada } \\
\text { psicomotora e epistaxe. }\end{array}$ & $\begin{array}{l}\text { Redução mais lenta da pressão arterial avaliada em até } \\
24 \text { horas. }\end{array}$ \\
\hline
\end{tabular}

Abordagem propedêutica do suporte diagnóstico ideais nas urgências e emergências hipertensivas.

\begin{tabular}{|c|c|c|}
\hline Entrevista & Exame Físico & Exames Complementares \\
\hline
\end{tabular}


Situações que caracterizam as urgências e emergências hipertensivas.

\begin{tabular}{|l|l|}
\hline \multicolumn{1}{|c|}{ Emergências } & \multicolumn{1}{|c|}{ Urgências } \\
\hline Encefalopatia hipertensiva & Angina Instável \\
\hline Hemorragia cerebral & Pré-eclâmpsia \\
\hline Dissecção aguda de aorta & Anticoagulação \\
\hline Infarto agudo do miocárdio & Intoxicação por cocaína ou anfetamina \\
\hline Insuficiência cardíaca refratária & Pré e pós-operatório \\
\hline Edema agudo de pulmão & Transplante renal \\
\hline Insuficiência renal & $\begin{array}{l}\text { Rebote hipertensivo após suspensão súbita de clonidina } \\
\text { ou outros anti-hipertensivos }\end{array}$ \\
\hline Eclâmpsia & \\
\hline
\end{tabular}

Drogas endovenosas indicadas nas emergências hipertensivas

\begin{tabular}{|l|l|l|l|l|l|l|}
\hline \multicolumn{1}{|c|}{ Classe } & \multicolumn{1}{|c|}{ Droga } & Dose & Inicio & Duração & Indicação Formal & $\begin{array}{l}\text { Efeitos adversos e } \\
\text { precauções. }\end{array}$ \\
\hline Vasodilatadores & $\begin{array}{l}\text { Nitroprussiato } \\
\text { (Nipride@) }\end{array}$ & $\begin{array}{l}0,25 \mathrm{mcg} / \\
\mathrm{kg} / \mathrm{min}\end{array}$ & Imediato & $2-5 \mathrm{~min}$ & $\begin{array}{l}\text { Náuseas, vômito, } \\
\text { intoxicação por } \\
\text { cianeto. Cuidado na } \\
\text { insuficiência renal, } \\
\text { hipotensão severa. }\end{array}$ \\
\hline Diuréticos & Furosemida & $20-40 \mathrm{mg}$ & $5-15 \mathrm{~min}$ & $2-3 \mathrm{~h}$ & $\begin{array}{l}\text { Edema Agudo de } \\
\text { Pulmão }\end{array}$ & $\begin{array}{l}\text { Depleção de volume } \\
\text { e hipocalemia. }\end{array}$ \\
\hline Inibidores Adre- \\
nérgicos
\end{tabular}

